\title{
Analysis of repeated 24-core saturation prostate biopsy: Inverse association between asymptomatic histological inflammation and prostate cancer detection
}

\author{
TOMONORI KATO, AKIRA KOMIYA, AKIHIRO MORII, HIROAKI IIDA, TAKATOSHI ITO and HIDEKI FUSE \\ Department of Urology, Graduate School of Medicine and Pharmaceutical Sciences for Research, \\ University of Toyama, Toyama 930-0194, Japan
}

Received February 9, 2015; Accepted April 5, 2016

DOI: $10.3892 / 01.2016 .4693$

\begin{abstract}
Saturation prostate biopsy protocols have been developed to improve the prostate cancer ( $\mathrm{PCa}$ ) detection rate, particularly in the setting of repeat biopsies. The present study attempted to clarify the association between PCa detection and various risk factors in repeat saturation biopsies. A retrospective analysis was conducted on 78 Japanese patients for whom findings had caused suspicion of PCa despite previous negative prostate biopsies, and who consecutively underwent a 24-core transperineal repeat biopsy at Toyama University Hospital (Toyama, Japan). PCa was confirmed histologically in 16 of the 78 patients (20.5\%). A univariate analysis revealed that the prostate-specific antigen (PSA) level at repeat biopsy was higher $(\mathrm{P}<0.01)$, the fPSA/tPSA ratio was lower $(\mathrm{P}=0.04)$, the total prostate volume was smaller $(\mathrm{P}=0.01)$ and the PSA density was higher $(\mathrm{P}<0.01)$ in $\mathrm{PCa}$ patients than in patients with benign prostatic disease (BPD). Histological inflammation was more frequently observed in BPD patients than in PCa patients $(\mathrm{P}<0.01)$. A multivariate analysis revealed that histological inflammation was the only independent predictor of the presence of a malignant lesion on repeat biopsy (odds ratio, $0.027 ; \mathrm{P}=0.01)$. It must be considered that inflammation may cause a PSA increase in some patients with a negative initial biopsy, leading to unnecessary repeat biopsy.
\end{abstract}

Correspondence to: Dr Akira Komiya, Department of Urology, Graduate School of Medicine and Pharmaceutical Sciences for Research, University of Toyama, 2630 Sugitani, Toyama 930-0194, Japan

E-mail: komiya@med.u-toyama.ac.jp

Abbreviations: $\mathrm{PCa}$, prostate cancer; $\mathrm{BPD}$, benign prostatic disease; PSA, prostate-specific antigen; PSAD, PSA density; PSAV, PSA velocity; DRE, digital rectal examination; MRI, magnetic resonance imaging; TRUS, transrectal ultrasonography; ASAP, atypical small acinar proliferation; HGPIN, high-grade prostatic intraepithelial neoplasia; RRP, radical retropubic prostatectomy; SD, standard deviation

Key words: prostate cancer, saturation biopsy, repeat biopsy, transperineal biopsy, histological inflammation

\section{Introduction}

With the introduction of the serum prostate-specific antigen (PSA) assay in combination with digital rectal examination (DRE) to screen for prostate cancer (PCa), the number of patients undergoing subsequent transrectal ultrasonography (TRUS)-guided needle biopsy has markedly increased (1). In addition, the systematic sextant biopsy technique has become the standard method for prostate biopsy, which has resulted in a significant improvement in the accuracy of PCa diagnosis (2). However, a number of studies have suggested that sextant biopsy may underestimate the presence of malignancy $(3,4)$. Thus, there has been increasing interest in defining more accurate prostate biopsy strategies in order to improve the PCa detection rate. The benefits of increasing the number of biopsy cores and/or expanding the number of regions sampled have been demonstrated previously $(5,6)$; however, it has also been suggested that a 10 - to 12 -core initial biopsy may miss $\mathrm{PCa}$ in almost a third of cases $(7,8)$. Saturation prostate biopsy, which consists of $\geq 20$ cores, has been developed to improve the detection rate of $\mathrm{PCa}$ in the context of repeat biopsies, radically altering the general concept of prostate biopsy (9-12).

The decision to conduct a prostate biopsy predominantly depends on the PSA value, which often generates false positive results as this value may be increased by inflammation (13). Our group previously reported that histological prostatic inflammation significantly correlated with the serum PSA level and a negative initial biopsy result (14). Consequently, we hypothesized that asymptomatic prostatic inflammation may cause a persistent PSA increase in patients with a negative initial biopsy, leading to repeat biopsy.

The present study attempted to clarify the association between the risk factors for PCa and PCa detection in a repeat biopsy with a 24-core saturation protocol. Repeat biopsy specimens were evaluated for evidence of histological inflammation by examining the leukocytes as one of the risk factors for PCa.

\section{Materials and methods}

Patients. Commencing in December 2010, 24-core repeat biopsies were performed in patients for whom findings aroused 
suspicion of PCa despite previous negative prostate biopsies. Such patients included those with any of the following indications for repeat biopsy: PSA >10 ng/ml; free-to-total PSA (fPSA/tPSA) ratio < $15 \%$; PSA velocity (PSAV) $>0.75 \mathrm{ng} / \mathrm{ml} /$ year; abnormal findings on DRE, magnetic resonance imaging (MRI) or TRUS; or atypical small acinar proliferation (ASAP) in the initial biopsy specimens.

The analysis included 78 Japanese patients whose initial biopsy was negative and who consecutively underwent 24-core prostate transperineal needle biopsy as a repeat biopsy between December 2010 and November 2013 at Toyama University Hospital (Toyama, Japan). All patients underwent TRUS to calculate the whole prostate volume. Prostate volume (ml) was calculated using the prostate ellipsoid formula (width $\mathrm{x}$ length $\mathrm{x}$ height $\mathrm{x}$ 0.523). PSA density (PSAD) was calculated by dividing the total PSA $(\mathrm{ng} / \mathrm{ml})$ by the prostate volume. The data were retrospectively analyzed. The institutional review board of the University of Toyama (Toyama, Japan) approved the study (\#26-34). Due to the retrospective nature of the study, written informed consent was waived. The study conformed to the principles outlined in the Declaration of Helsinki (15).

TRUS-guided systematic biopsy. Biopsies were performed with the patient in a dorsal lithotomy position under spinal anesthesia. TRUS was performed using the Aloka Prosound Alpha 5 SV Ultrasound system and a 5.0/7.5 MHz biplanar probe (Hitachi Aloka Medical, Ltd., Tokyo, Japan). Transperineal biopsies of the prostate were obtained with an 18-gauge spring-loaded biopsy gun ( Bard $^{\circledR}$ Max-Core ${ }^{\circledR}$ Disposable Core Biopsy Instrument; C. R. Bard, Inc., Tempe, AZ, USA) under TRUS guidance. Biopsy cores were taken from 24 biopsy locations based on a standardized biopsy scheme (Fig. 1) described by Abdollah et al (9), with some modification. Biopsy cores of odd numbers were taken from the apex, whereas cores of even numbers were taken from the base of the prostate. Urethral catheters were routinely inserted following the procedures. In the majority of cases without severe adverse effects, the urethral catheters were removed on the first day and patients were discharged within three days subsequent to the procedure. All biopsy samples were evaluated by the Department of Pathology at Toyama University Hospital using the Gleason scoring system (16). These findings were morphologically evaluated on hematoxylin and eosin stained tissue sections. Histological inflammation of the prostate was defined as infiltration of prostatic biopsy specimens by inflammatory cells, lymphocytes, plasma cells and/or histiocytes (17).

Statistical analysis. Data are presented as the mean \pm standard deviation (SD). Variables from the different groups were compared by the Mann-Whitney U-test or Student's $t$-test using StatView version 5 software (SAS Institute, Cary, NC, USA). $\mathrm{P}<0.05$ was considered to indicate a statistically significant difference. Independent factors predicting a positive repeat biopsy were identified by a logistic regression analysis.

\section{Results}

Patient characteristics. Tables I and II list the characteristics of all 78 patients who underwent repeat prostate biopsies
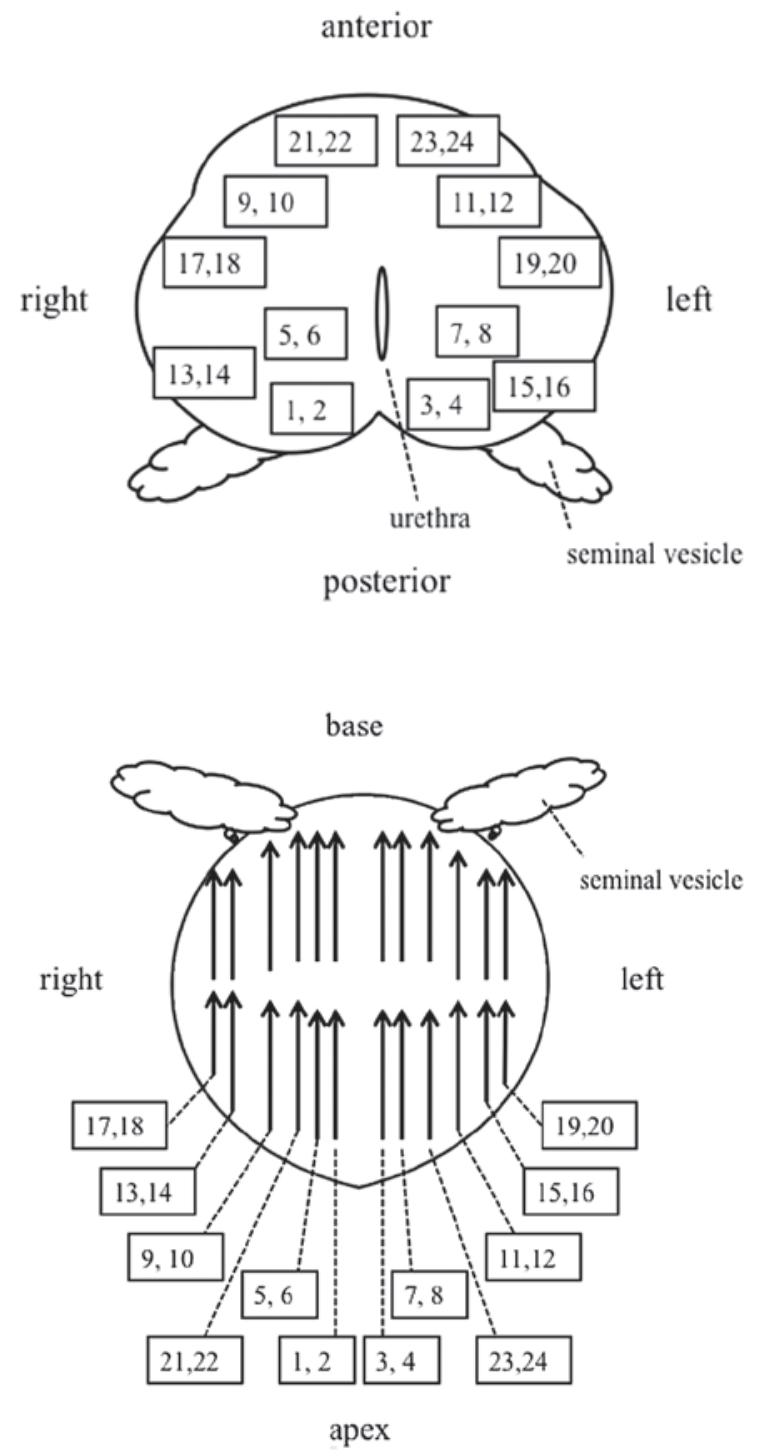

Figure 1. Biopsy cores were taken from 24 locations based on a standardized biopsy scheme (Abdollah et al) (9). The biopsy cores of odd numbers were taken from the apex, whereas cores of even numbers were taken from the base of the prostate.

following previous negative biopsies according to the indications described. The indications for biopsies were as follows: a PSA value $>10 \mathrm{ng} / \mathrm{ml}$ in $43.6 \%$ of cases; a fPSA/tPSA ratio $<15 \%$ in $51.3 \%$; a PSAV $>0.75 \mathrm{ng} / \mathrm{ml} /$ year in $53.8 \%$; abnormal findings on DRE in $10.2 \%$, TRUS in $26.9 \%$ or MRI in $44.1 \%$; and ASAP in previous biopsy specimens in $18.4 \%$. Of the included patients, $19(24.4 \%)$ had a single indication, whilst the remaining $59(75.6 \%)$ had multiple indications. The mean age at the time of repeat biopsy was $68.5 \pm 7.0$ years and the mean PSA level prior to repeat biopsy was $12.4 \pm 10.7 \mathrm{ng} / \mathrm{ml}$. The mean number of previous TRUS-guided needle biopsies was 1.6 \pm 1.0 (range, 1-6). Collectively, the 78 patients had experienced a total of 124 previous biopsies, with mean of $10.7 \pm 3.5$ cores per patient (range, 6-24 cores), sampled via 82 transrectal (66.1\%), 13 transperineal (10.4\%) and 29 unknown (23.3\%) routes.

Cancer detection. PCa was confirmed histologically in 16 of the 78 patients $(20.5 \%)$, and benign prostatic disease (BPD) was diagnosed in the remaining 62 patients $(79.5 \%)$ based 
Table I. Characteristics of 78 patients who underwent repeat prostate biopsies following previous negative biopsies.

\begin{tabular}{l} 
Parameter \\
\hline Age at diagnosis, years \\
$50-59$ \\
$60-69$ \\
$70-79$ \\
$80+$ \\
Number of previous \\
negative biopsies \\
1 \\
2 \\
3 \\
$\geq 4$
\end{tabular}

Serum PSA value at repeat biopsy, $\mathrm{ng} / \mathrm{ml}$

$\begin{array}{lr}3.0-10.0 & 44 \\ 10.1-20.0 & 24 \\ 20.1-50.0 & 9 \\ 50.1-100.0 & 1 \\ \text { fPSA/tPSA ratio, \% } & \\ 5.0-15.0 & 40 \\ 15.1-30.0 & 22 \\ 30.1-50.0 & 4 \\ \text { Not measured } & 12\end{array}$

Prostate volume, $\mathrm{ml}$

20.0-40.0

$40.1-60.0$

$60.1-100.0$

$>100.0$

PSA density, $\mathrm{ng} / \mathrm{ml} / \mathrm{ml}$

0.050-0.150

$0.151-0.50$

$>0.50$

PSA velocity, ng/ml/year

$<0$

27

33

4

\section{0}

17

7
7
4

mean \pm SD

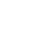

$68.5 \pm 7.0$

$1.60 \pm 1.03$

1

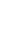

$47.3 \pm 19.0$

$12.4 \pm 10.7$

on the repeat biopsies. Table II shows the clinical parameters of the PCa and BPD patients. A univariate analysis revealed that the PSA value at repeat biopsy, the fPSA/tPSA ratio, the prostate volume and the PSAD were significantly associated with the repeat biopsy outcome. PSA at repeat biopsy was higher $(\mathrm{P}<0.01)$, fPSA/tPSA ratio was lower $(\mathrm{P}=0.04)$, total prostate volume was smaller $(\mathrm{P}=0.01)$ and $\mathrm{PSAD}$ was higher $(\mathrm{P}<0.01)$ in patients with $\mathrm{PCa}$ compared with BPD patients. Furthermore, histological inflammation was more frequently observed in BPD patients than PCa patients $(\mathrm{P}<0.01)$. Unexpectedly, the differences between the $\mathrm{PCa}$ and $\mathrm{BPD}$ patients were not significant for abnormal findings on DRE, MRI or TRUS. The presence of ASAP in the initial biopsy specimens was also not associated with the outcome of the repeat biopsy.

Table III demonstrates results of the multivariate analysis of predictive variables for the presence of prostate cancer on repeat biopsy. The value of these factors as predictors of PCa detection on repeat biopsy was subsequently analyzed using a logistic regression analysis. The variables entered into the model were PSA at repeat biopsy, fPSA/tPSA ratio, prostate volume, PSAD and presence of histological inflammation. Among these factors, histological inflammation was the only independent predictor for the presence of a malignant lesion on repeat biopsy (odds ratio, $0.027 ; \mathrm{P}=0.01$ ).

$0.63 \pm 16.27$ Characteristics of prostate cancer patients diagnosed by repeat biopsies. Table IV lists the characteristics of all 16 patients diagnosed with $\mathrm{PCa}$. Of these 16 patients, $14(87.5 \%)$ had localized disease and $2(12.5 \%)$ had metastasis. The clinical stages were T1c, T2a and T2b in $4(25 \%)$, $10(62.5 \%)$ and $2(12.5 \%)$ patients, respectively. The Gleason scores were $3+3,3+4$ and $\geq 4+4$ in $6(37.5 \%), 3(18.8 \%)$ and $7(43.8 \%)$ patients, respectively.

Of the 16 cases, 2 were compatible with the widely used criteria for predicting clinically insignificant $\mathrm{PCa}$, consisting of T1c, PSA $\leq 10 \mathrm{ng} / \mathrm{ml}$, Gleason score $\leq 6$ and $<50 \%$ cancer involvement in any core (18). Of these, 1 patient was subsequently determined to have significant cancer. The PSA level, the clinical stage, and the Gleason score of this patient were $5.73 \mathrm{ng} / \mathrm{ml}$, T1cN0M0, 3+3, respectively, and 3/24 cores were positive for cancer. When the patient was subsequently treated with radical retropubic prostatectomy (RRP), pT2c significant disease and a Gleason score of $4+5$ in the prostatectomy specimen were determined. By contrast, the other patient was confirmed to have clinically insignificant cancer. The detailed 
Table II. Clinical parameters of the PCa and BPD patients.

\begin{tabular}{|c|c|c|c|c|}
\hline Parameters & All $(n=78)$ & $\mathrm{PCa}(\mathrm{n}=16)$ & $\mathrm{BPD}(\mathrm{n}=62)$ & P-value \\
\hline Age at diagnosis, years ${ }^{\mathrm{a}}$ & $68.5 \pm 7.0$ & $69.5 \pm 7.7$ & $68.2 \pm 6.8$ & 0.54 \\
\hline Number of previous biopsies ${ }^{a}$ & $1.60 \pm 1.03$ & $1.62 \pm 1.31$ & $1.59 \pm 0.96$ & 0.92 \\
\hline Serum PSA value at repeat biopsy, $\mathrm{ng} / \mathrm{ml}^{\mathrm{a}}$ & $12.4 \pm 10.7$ & $18.9 \pm 18.2$ & $10.8 \pm 7.0$ & $<0.01$ \\
\hline fPSA/tPSA ratio, $\%^{\mathrm{a}}$ & $15.6 \pm 9.3$ & $11.1 \pm 5.2$ & $16.8 \pm 9.9$ & 0.04 \\
\hline Prostate volume, $\mathrm{ml}^{\mathrm{a}}$ & $47.3 \pm 19.0$ & $36.5 \pm 17.3$ & $50.0 \pm 18.5$ & 0.01 \\
\hline PSA density, $\mathrm{ng} / \mathrm{ml} / \mathrm{ml}^{\mathrm{a}}$ & $0.32 \pm 0.41$ & $0.62 \pm 0.71$ & $0.24 \pm 0.23$ & $<0.01$ \\
\hline PSA velocity, ng/ml/year ${ }^{\mathrm{a}}$ & $0.63 \pm 16.27$ & $4.62 \pm 5.81$ & $50.0 \pm 17.9$ & 0.27 \\
\hline Abnormal findings on $\mathrm{DRE}^{\mathrm{b}}$ & $10.2 \%(8 / 78)$ & $12.5 \%(2 / 16)$ & $9.6 \%(6 / 62)$ & 0.76 \\
\hline Abnormal findings on TRUS ${ }^{b}$ & $26.9 \%(21 / 78)$ & $31.2 \%(5 / 16)$ & $25.8 \%(16 / 62)$ & 0.66 \\
\hline Abnormal findings on $\mathrm{MRI}^{\mathrm{b}}$ & $44.1 \%(34 / 77)$ & $56.3 \%(9 / 16)$ & $40.9 \%(25 / 61)$ & 0.28 \\
\hline ASAP in initial biopsy specimens ${ }^{\text {b }}$ & $14.1 \%(11 / 76)$ & $18.8 \%(3 / 16)$ & $12.9 \%(8 / 62)$ & 0.55 \\
\hline Histological inflammation ${ }^{\mathrm{b}}$ & $47.4 \%(37 / 78)$ & $6.3 \%(1 / 16)$ & $58.0 \%(36 / 62)$ & $<0.01$ \\
\hline
\end{tabular}

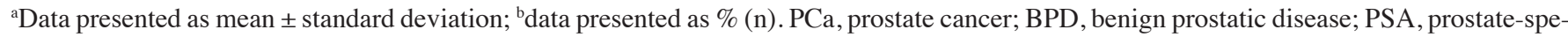
cific antigen; fPSA/tPSA, free-to-total PSA; DRE, digital rectal examination; TRUS, transrectal ultrasonography; MRI, magnetic resonance imaging; ASAP, atypical small acinar proliferation.

Table III. Results of the multivariate analysis of predictive variables for the presence of prostate cancer on repeat biopsy.

\begin{tabular}{lccc}
\hline Factor & P-value & Relative hazard ratio & $95 \%$ confidence interval \\
\hline Serum PSA value at repeat biopsy & 0.64 & 0.87 & $0.50-1.51$ \\
fPSA/tPSA ratio & 0.34 & 0.93 & $0.82-1.07$ \\
Prostate volume & 0.80 & 0.98 & $0.86-1.12$ \\
PSA density & 0.47 & 571 & $1.5 \times 10^{-5}-2.1 \times 10^{10}$ \\
Histological inflammation & 0.01 & 0.027 & $0.001-0.54$ \\
\hline
\end{tabular}

PSA, prostate specific antigen; fPSA/tPSA, free-to-total PSA.

clinical findings of this patient were as follows: PSA at repeat biopsy, $4.48 \mathrm{ng} / \mathrm{ml}$; clinical stage, T1cN0M0; Gleason score, $3+3$; and $1 / 24$ cores positive for cancer. This patient subsequently underwent RRP; however, the pathological stage was pT0.

Adverse effects. Major complications associated with biopsy that required subsequent inpatient intervention occurred in 3 patients (3.8\%): 2 patients experienced urinary retention requiring temporary catheterization; the other patient experienced severe septicaemia with Escherichia coli that required intravenous antibiotic administration, and this patient made a full recovery two weeks after the procedure.

\section{Discussion}

To date, PSA measurement has been the most valuable criterion upon which to base a diagnosis of $\mathrm{PCa}$ (19). The benefits of increasing the number of biopsy cores and/or expanding the number of regions sampled have been demonstrated in several studies. Pepe et al (5) compared 12-core with 18-core biopsies, and identified significant differences in the detection rates, which were determined to be 35 and $51 \%$, respectively. Ficarra et al (6) demonstrated a correlation between a detection rate and a core count. Initial biopsy protocols have been gradually extended to improve the detection rate through more representative sampling of all areas in the prostate $(5,6)$.

Repeat saturation biopsies have also been developed in an effort to reduce the high false-negative rate of initial biopsies. Several studies introduced modified techniques for repeat biopsy involving an increased number of cores in order to sample the entire gland. Merrick et al (10) demonstrated a positive correlation between the detection rate and core count in a 50-core repeat biopsy series. In a study by Borboroglu et al (11), an average of 22.5 cores was obtained (depending on prostate size) in patients with previous negative sextant biopsies, and cancer was identified in $30 \%$ of patients. Similarly, Stewart et al (12) obtained an average of 23 cores throughout the whole prostate of patients who had previously had negative sextant biopsies, and cancer was detected in 34\% of cases.

To date, there are no established procedures for repeat saturation biopsy. Abdollah et al (9) evaluated 332 patients who underwent a transrectal 24-core repeat biopsy compared with 140 who underwent a 24-core transperineal biopsy, and concluded that transperineal and transrectal saturation biopsies have a similar $\mathrm{PCa}$ detection rate in patients undergoing 
Table IV. Characteristics of 16 patients diagnosed with prostate cancer by repeat biopsy.

\begin{tabular}{lr} 
Parameters & $\mathrm{n}$ \\
\hline Serum PSA value at repeat biopsy, ng/ml & \\
$>3-10$ & 6 \\
$>10-20$ & 5 \\
$>20-50$ & 4 \\
$>50-100$ & 1 \\
Clinical T stage & \\
T1c & 4 \\
T2a & 10 \\
T2b & 2 \\
Clinical N/M stage & 14 \\
N0M0 & 1 \\
N1M0 & 1 \\
N0M1 & \\
Gleason score & \\
$3+3$ & 6 \\
$3+4$ & 3 \\
$>4+4$ & 7 \\
Number of positive cores & 2 \\
1-2 & 6 \\
5-4 & \\
$10-15$ & 6 \\
\hline
\end{tabular}

PSA, prostate-specific antigen; $\mathrm{T}$, tumor; $\mathrm{N}$, node; $\mathrm{M}$, metastasis.

a repeated saturation biopsy. On the other hand, a number of studies have demonstrated that tumors subsequently identified upon repeat biopsy are most frequently found in the anterior prostate and/or transition zone $(20,21)$. In the present study, a transperineal route was used for repeat biopsy as the majority of the patients had undergone initial biopsies via the transrectal approach. Geometric considerations dictate that the apex and the anterior region of the prostate are better sampled via the transperineal route, while the base is better sampled via the transrectal approach. However, in the current study, the sites in which the cancer was detected were distributed uniformly, and there did not seem to be any trends in the sampling site (data not shown).

The detection of clinically insignificant $\mathrm{PCa}$ is an inevitable risk of repeat biopsy (14). The majority of patients in the current study were considered to have significant cancer. In particular, 2 patients (12.5\%), whose cancer was not detected in the initial biopsy, had metastatic disease. Thus, the present saturation protocol has demonstrated generally favorable results. However, 1 patient (6.25\%) was considered to have insignificant cancer in the current study. It is supposed that clinically insignificant disease is more likely to be detected with more extensive sampling, which is why extensive sampling remains controversial. A study by Zaytoun et al (22) suggested that almost a third of PCa detected in the repeat saturation biopsy was clinically insignificant. Conversely, a number of reports have demonstrated that saturation repeat biopsy may not increase the detection of clinically insignificant tumors $(23,24)$.

Discriminating between PCa and BPD is difficult, particularly in patients with initial negative biopsies $(25,26)$. The serum PSA level at repeat biopsy is one of the strongest predictors of a positive repeat biopsy $(25,26)$. However, it is generally recognized that PSA lacks specificity for PCa detection (14). Several clinically available PSA-associated factors, including the free-to-total PSA ratio, PSAD and PSAV, have been examined in an attempt to improve the efficiency of repeat biopsies; however, the value of these parameters remains the subject of considerable debate.

The significance of the prostate volume for the detection of PCa is thought to differ between the initial and repeat biopsies (27-29). For initial biopsies, previous studies have reported a decreasing probability of detecting cancer when the same number of biopsy cores is taken from glands of increasing size, indicating a potential benefit of increasing the number of core samples in larger glands (27). In addition, several studies have demonstrated an inverse correlation between the PCa detection rate in repeat saturation biopsies and the prostate volume; that is, there was a lower PCa detection rate in large prostates compared with smaller glands $(28,29)$.

Consistent with previous reports $(9,11,22,26,28,30)$, the current study demonstrated that a higher PSA level at repeat biopsy, lower prostate volume and elevated PSAD, were associated with a higher rate of $\mathrm{PCa}$ detection. In the context of repeat biopsy, intensive saturation biopsies should be recommended in patients with a smaller prostate volume or increased PSAD.

DRE is indispensable for PCa screening, and is a meaningful indication for an initial biopsy (14). Abnormal findings on MRI and TRUS are also significant predictors of PCa, particularly in the initial biopsy setting (31). By contrast, in the repeat biopsy setting, the present study demonstrated that the incidence of abnormal findings on DRE, TRUS and/or MRI in patients with PCa was not significantly higher than that in patients with no evidence of cancer. We hypothesize that this was due to a selection bias, i.e. patients with well-visualized cancers had already been diagnosed in the initial biopsies, and a biased population therefore remained in the repeat biopsy setting.

Previously, the presence of high-grade prostatic intraepithelial neoplasia (HGPIN) and/or ASAP was demonstrated to be a useful predictor of the repeat biopsy outcome $(28,32)$. The role of HGPIN as a precursor of PCa remains controversial. According to recent guidelines, a HGPIN diagnosis no longer represents an indication for immediate repeat biopsy (33). By contrast, ASAP indicates the presence of suspicious glands with insufficient cytological or architectural atypia for a definitive diagnosis of prostatic adenocarcinoma (30). In the present study, the incidence of PCa was not significantly higher in patients with ASAP. This may have been due to the limited number of patients with ASAP in this cohort, and so these findings are not conclusive. Further studies are required to clarify the prognostic value of the presence of ASAP.

Serum PSA levels may be increased not only in patients with PCa, but also in those with non-malignant conditions, including benign prostatic hyperplasia, prostatic manipulation and even asymptomatic and chronic prostatitis $(19,34)$. 
Prostatic inflammation is detected in some biopsy specimens, and subclinical prostatitis is known to cause increases in the serum PSA level (35-37). Morote et al (13) retrospectively studied 284 patients with no evidence of cancer on sextant ultrasound-guided biopsies. Benign tissue without inflammation was detected in $23.2 \%$ of the patients, whilst $68.3 \%$ had chronic prostatitis and $8.4 \%$ had acute prostatitis, suggesting that prostatic inflammation is common in specimens of BPD. Our group previously reported that histological prostatic inflammation was significantly correlated with the serum PSA level and a negative biopsy result (14). Similarly, Terakawa et al (38) reported that histological inflammation in initial biopsy specimens was associated with a negative initial biopsy outcome in men with a serum PSA level of 10-50 ng/ml.

In the current study, histological inflammation was found to be independently associated with a lower risk of PCa, even on repeat biopsy. Asymptomatic inflammation may cause increased PSA in some men, leading to repeat prostate biopsy (14). A more selective repeat biopsy strategy would help avoid unnecessary repeat biopsies in men with an increased PSA level.

The limitations of this study include its retrospective design, its being performed at a single center using a single arm, and the relatively small number of patients. The results may have been biased by the patient selection for repeat biopsy. The probability of detecting clinically insignificant $\mathrm{PCa}$ is still uncertain based on this study due to the limited number of patients; further prospective studies with large populations are necessary in order to clarify this issue. Nevertheless, even with these limitations, the current results suggest that saturation repeat biopsy may be effective in patients for whom $\mathrm{PCa}$ is persistently suspected despite a negative initial biopsy.

Although numerous trials have been published, the number and most appropriate location of the cores, in addition to the timing and the criteria to perform repeat biopsy, remains matters of debate. However, saturation biopsy appears to be effective in patients with a persistent suspicion of $\mathrm{PCa}$ following a negative initial biopsy. A relatively small prostate volume in a patient with an elevated PSA level (i.e. a high PSAD) may be a useful indicator of the results of a repeat saturation biopsy. Furthermore, patients with asymptomatic inflammation appear to have a lower risk of PCa. It should be considered that inflammation may cause persistent elevated PSA in patients with a negative initial biopsy, leading to unnecessary repeat biopsy.

\section{Acknowledgements}

The authors would like to thank Professor Joji Imura of the Department of Diagnostic Pathology, Graduate School of Medicine and Pharmaceutical Sciences for Research, University of Toyama, for his valuable advice and suggestions with regard to pathological findings.

\section{References}

1. Ouyang RC, Kenwright DN, Nacey JN and Delahunt B: The presence of atypical small acinar proliferation in prostate needle biopsy is predictive of carcinoma on subsequent biopsy. BJU Int 87: 70-74, 2001.

2. Hodge KK, McNeal JE, Terris MK and Stamey TA: Random systematic versus directed ultrasound guided transrectal core biopsies of the prostate. J Urol 142: 71-75, 1989.
3. Rabbani F, Stroumbakis N, Kava BR, Cookson MS and Fair WR: Incidence and clinical significance of false-negative sextant prostate biopsies. J Urol 159: 1247-1250, 1998.

4. Norberg M, Egevad L, Holmberg L, Sparèn P, Norlèn BJ and Busch C: The sextant protocol for ultrasound-guided core biopsies of the prostate underestimates the presence of cancer. Urology 50: 562-566, 1997.

5. Pepe P and Aragona F: Prostate needle biopsy: 12 vs. 18 cores - is it necessary? Urol Int 74: 19-22, 2005.

6. Ficarra V, Novella G, Novara G, Galfano A, Pea M, Martignoni G and Artibani W: The potential impact of prostate volume in the planning of optimal number of cores in the systematic transperineal prostate biopsy. Eur Urol 48: 932-937, 2005.

7. Schröder FH, Hugosson J, Roobol MJ, Tammela TL, Ciatto S, Nelen V, Kwiatkowski M, Lujan M, Lilja H, Zappa M, et al: Screening and prostate-cancer mortality in a randomized European study. N Engl J Med 360: 1320-1328, 2009.

8. Andriole GL, Crawford ED, Grubb RL III, Buys SS, Chia D, Church TR, Fouad MN, Gelmann EP, Kvale PA, Reding DJ, et al: Mortality results from a randomized prostate-cancer screening trial. N Engl J Med 360: 1310-1319, 2009.

9. Abdollah F, Novara G, Briganti A, Scattoni V, Raber M, Roscigno M, Suardi N, Gallina A, Artibani W, Ficarra V, et al: Transrectal versus transperineal saturation rebiopsy of the prostate: Is there a difference in cancer detection rate? Urology 77: 921-925, 2011.

10. Merrick GS, Gutman S, Andreini H, Taubenslag W, Lindert DL, Curtis R, Adamovich E, Anderson R, Allen Z, Butler W and Wallner K: Prostate cancer distribution in patients diagnosed by transperineal template-guided saturation biopsy. Eur Urol 52: 715-723, 2007.

11. Borboroglu PG, Corner SW, Riffenburgh RH and Amling CL: Extensive repeat transrectal ultrasound-guided prostate biopsy in patient with previous benign sextant biopsies. J Urol 163: 158-162, 2000.

12. Stewart CS, Leibovich BC, Weaver AL and Lieber MM: Prostate cancer diagnosis using a saturation needle biopsy technique after previous negative sextant biopsies. J Urol 166: 86-92, 2001.

13. Morote J, Lopez M, Encabo G and de Torres IM: Effect of inflammation and benign prostatic enlargement on total and percent free serum prostatic specific antigen. Eur Urol 37: 537-540, 2000.

14. Kato T, Suzuki H, Komiya A, Imamoto T, Naya Y, Tobe T and Ichikawa T: Clinical significance of urinary white blood cell count and serum C-reactive protein level for detection of non-palpable prostate cancer. Int J Urol 13: 915-919, 2006.

15. Hellmann F, Verdi M, Schlemper BR Jr and Caponi S: 50th anniversary of the Declaration of Helsinki: The double standard was introduced. Arch Med Res 45: 600-601, 2014.

16. Epstein JI, Allsbrook WC Jr, Amin MB and Egevad LL; ISUP Grading Committee: The 2005 International Society of Urological Pathology (ISUP) Consensus Conference on Gleason Grading of prostatic carcinoma. Am J Surg Pathol 29: 1228-1242, 2005.

17. Krieger JN, Nyberg L Jr and Nickel JC: NIH consensus definition and classification of prostatitis. JAMA 282: 236-237, 1999.

18. Tosoian JJ, Trock BJ, Landis P, Feng Z, Epstein JI, Partin AW, Walsh PC and Carter HB: Active surveillance program for prostate cancer: An update of the Johns Hopkins experience. J Clin Oncol 29: 2185-2190, 2011.

19. Oesterling JE: Prostate specific antigen: A critical assessment of the most useful tumor marker for adenocarcinoma of the prostate. J Urol 145: 907-923, 1991.

20. Kawakami S, Kihara K, Fujii Y, Masuda H, Kobayashi T and Kageyama Y: Transrectal ultrasound-guided transperineal 14-core systematic biopsy detects apico-anterior cancer foci of T1c prostate cancer. Int J Urol 11: 613-618, 2004.

21. Shannon BA, Mc Neal JE and Cohen RJ: Transition zone carcinoma of the prostate gland: A common indolent tumor type that occasionally manifests aggressive behaviour. Pathology 35: 467-471, 2003

22. Zaytoun OM, Moussa AS, Gao T, Fareed K and Jones JS: Office-based transrectal saturation biopsy improves prostate cancer detection compared to extended biopsy in the repeat biopsy population. J Urol 186: 850-854, 2011.

23. Bastian PJ, Mangold LA, Epstein JI and Partin AW: Characteristics of insignificant clinical T1c prostate tumors. A contemporary analysis. Cancer 101: 2001-2005, 2004.

24. Epstein JI, Sanderson H, Carter HB and Scharfstein DO: Utility of saturation biopsy to predict insignificant cancer at radical prostatectomy. Urology 66: 356-360, 2005. 
25. Gann PH, Fought A, Deaton R, Catalona WJ and Vonesh E: Risk factors for prostate cancer detection after a negative biopsy: A novel multivariable longitudinal approach. J Clin Oncol 28 $1714-1720,2010$

26. Djavan B, Fong YK, Ravery V, Remzi M, Horninger W, Susani M Kreuzer S, Boccon-Gibod L, Bartsch G and Marberger M: Are repeat biopsies required in men with PSA levels $<$ or $=4 \mathrm{ng} / \mathrm{ml}$ ? A multi-institutional prospective European study. Eur Urol 47: 38-44, 2005.

27. Brawer MK: The influence of prostate volume on prostate cancer detection. Eur Urol Suppl 1: 35-39, 2002.

28. Campos-Fernandes JL, Bastien L, Nicolaiew N, Robert G, Terry S, Vacherot F, Salomon L, Allory Y, Vordos D, Hoznek A, et al: Prostate cancer detection rate in patients with repeated extended 21-sample needle biopsy. Eur Urol 55: 600-606, 2009.

29. Sajadi KP, Kim T, Terris MK, Brown JA and Lewis RW: High yield of saturation prostate biopsy for patients with previous negative biopsies and small prostates. Urology 70: 691-695, 2007.

30. Oderda $\mathrm{M}$ and Gontero P: High-grade prostatic intraepithelial neoplasia and atypical small acinar proliferation: Is repeat biopsy still necessary? BJU Int 104: 1554-1556, 2009.

31. Inahara M, Suzuki H, Nakamachi H, Kamiya N, Shimbo M Komiya A, Ueda T, Ichikawa T, Akakura K and Ito H: Clinical evaluation of transrectal power doppler imaging in the detection of prostate cancer. Int Urol Nephrol 36: 175-180, 2004.
32. Chan TY and Epstein JI: Follow-up of atypical prostate needle biopsies suspicious for cancer. Urology 53: 351-355, 1999.

33. Heidenreich A, Aus G, Bolla M, Joniau S, Matveev VB, Schmid HP and Zattoni F; European Association of Urology: EAU guidelines on prostate cancer. Eur Urol 53: 68-80, 2008.

34. Stamey TA, Yang N, Hay AR, McNeal JE, Freiha FS and Redwine E: Prostate-specific antigen as a serum marker for adenocarcinoma of the prostate. N Engl J Med 317: 909-916, 1987

35. Yaman O, Göğüş C, Tulunay O, Tokatli Z and Ozden E: Increased prostate-specific antigen in subclinical prostatitis: The role of aggressiveness and extension of inflammation. Urol Int 71: $160-164,2003$

36. Schatteman PH, Hoekx L, Wyndaele JJ, Jeuris W and Van Marck E: Inflammation in prostate biopsies of men without prostatic malignancy or clinical prostatitis: Correlation with total serum PSA and PSA density. Eur Urol 37: 404-412, 2000.

37. Kandirali E, Boran C, Serin E, Semercioz A and Metin A: Association of extent and aggressiveness of inflammation with serum PSA levels and PSA density in asymptomatic patients. Urology 70: 743-747, 2007.

38. Terakawa T, Miyake H, Kanomata N, Kumano M, Takenaka A and Fujisawao M: Inverse association between histologic inflammation in needle biopsy specimens and prostate cancer in men with serum PSA of 10-50 ng/ml. Urology 72: 1194-1197, 2008. 